\title{
A Robust Super-Tough Biodegradable Elastomer Engineered by Supramolecular Ionic Interactions
}

Hamed Daemi ${ }^{\mathrm{a}, \mathrm{b}}$, Sareh Rajabi-Zeleti ${ }^{\mathrm{b}}$, Haritz Sardon $^{\mathrm{c}}$, Mehdi Barikani ${ }^{\mathrm{a} *}$, Ali Khademhosseini $^{\mathrm{d}, \mathrm{e}, \mathrm{f}}$, Hossein Baharvand ${ }^{\mathrm{b}, \mathrm{g}, * *}$

${ }^{a}$ Department of Polyurethane, Iran Polymer \& Petrochemical Institute, Tehran, Iran

${ }^{b}$ Department of Stem Cells and Developmental Biology, Cell Science Research Center, Royan Institute, Tehran, Iran

${ }^{c}$ Polymat, University of the Basque Country UPV/EHU Joxe Mari Korta Center, Avda. Tolosa 72, 20018 San Sebastian, Spain

${ }^{d}$ Harvard-MIT Division of Health Sciences and Technology, Massachusetts Institute of Technology, Cambridge, MA

${ }^{e}$ Biomaterials Innovation Research Center, Biomedical Engineering Division, Department of Medicine, Brigham and Women's Hospital, Harvard Medical School, Cambridge, MA

${ }^{f}$ Wyss Institute for Biologically Inspired Engineering, Harvard University, Cambridge, MA

${ }^{g}$ Department of Developmental Biology, University of Science and Culture, ACECR, Tehran, Iran

\footnotetext{
* Corresponding author. Tel.: +98 21 48662427; fax: +98 2144580021.

E-mail address: M.Barikani@ippi.ac.ir (M. Barikani).

** Corresponding author. Tel.: +98 21 22306485; fax: +98 2123562507.

E-mail address: baharvand@ royaninstitute.org (H. Baharvand).
} 


\begin{abstract}
Alginate-based ionic polyurethanes (ASPUs) as mechanically tunable biomaterials with high strength and toughness in both dry and hydrated states are developed under metal-free conditions. The Young's modulus and tensile strength of ASPUs are tuned from 30 to 100 $\mathrm{MPa}$, and 20 to $50 \mathrm{MPa}$, respectively. Interestingly, the ASPUs exhibit a small hysteresis loop, minimal loss of tensile strength and minimal creep deformation after 100 repetitive cycles which makes them of use for engineering of load-bearing tissues. This is the first report that describes a linear PU can resist a large number of cyclic stresses without significant stretching. These bio-based elastomers engineered by ionic interactions are biocompatible and biodegradable. The ASPUs demonstrate a similar in vivo degradation rate compared to polycaprolactone (PCL). These biomaterials also demonstrate a rapid selfhealing and recovery after rupture, and have a linear biodegradation profile. Furthermore, histological examination of subcutaneous transplanted ASPUs after five months reveals low immunological response and low fibrosis.
\end{abstract}

Keywords: Supramolecular elastomer; Alginate; Load-bearing tissue; Self-healing; Biodegradation; Vascular tissue engineering 


\section{Introduction}

An ideal synthetic elastomer should possess tunable mechanical properties as well as appropriate cell attachment and tissue compatibility [1-3]. Furthermore, the hydration of a biomaterial as a scaffold is a critical parameter under dynamic in vivo conditions [4]. Biodegradable elastomers have been investigated for vascular tissue engineering applications because of their potential for controllable durability, tunable mechanical properties and desired cell affinity, and the ability to endure the repetitive cycles of physiologic loads [3]. It has been shown that most of natural and synthetic biomaterials cannot mimic the mechanical properties, including strength, initial stiffness, toughness, extensibility and recoverability of load-bearing soft tissues, due to the heterogeneity in their structures and/or lack of an efficient mechanism for energy dissipation [5]. Much research has been devoted to introduce new biodegradable polymers that possess improved mechanical properties. In this regard, photocrosslinkable tropoelastin (MeTro), poly(e-caprolactone) (PCL), poly(glycerolsebacate) (PGS) and citrate-based biodegradable elastomers (CABEs) demonstrate controllably mechanical properties [3,6-9]. However, the majority of these synthetic polymers are mechanically weak with a tensile strength of less than $5 \mathrm{MPa}$ in dry state compared to arteries, nerves or soft connective tissues such as human tendons, ligaments and articular cartilages which have tensile strengths of 10-50 MPa [3]. An additional challenge arises from the weaker mechanical properties of these materials when they are processed to fabricate porous scaffolds or used in physiological wet conditions. To address and improve this issue in mechanical properties, numerous chemical modification strategies have been reported [1], in which the chemical crosslinking is the most common procedure; however, this strategy drastically decelerates the rate of in vivo biodegradation. In addition to traditional improvements in chemical structure of biomaterials, the use of novel chemical strategies such as click chemistry, semi-interpenetrating polymer network (SIPN) structures and 
photocrosslinkable elastomers allow new approaches for the synthesis of biomaterials that contain tunable and improved properties for tissue engineering applications [10-12]. These synthetic strategies enrich the chemistry of biomaterials and improve their mechanical properties. However, the loss of biodegradability obtained from some linkages such as triazoles is an important disadvantage of their use in the synthesis of biodegradable materials. The unmet challenge is to find a simple, facile and modular synthetic strategy to balance the mechanical and biological properties of biomaterials. By keeping in mind these limitations, we designed a linear alginate-based polyurethane (APU) elastomer possibly having supramolecular interactions in the solid state with superior properties that makes it suitable for various applications including vascular tissue engineering.

To address the challenges associated with weak mechanical strength and hydration properties of biodegradable elastomers, polyurethanes (PUs) have been proposed as promising substitutes due to their high mechanical strength [13-18]. PUs display widely variable behaviors depending on their chemical and morphological makeup [19-21]. Therefore, a compromise must be found between biocompatibility and mechanical properties. In addition, for the preparation of aliphatic polyurethanes with high molecular weights, a toxic tin(II)based catalyst is commonly used which is not desirable for biological and biomedical applications [22,23].

As an expeditious and effective reversible chemical strategy, supramolecular ionic interactions are a promising substitute to enhance the mechanical properties of aliphatic PUs [24]. They are easily prepared by proton transfer reactions between multifunctional carboxylic acids and amines. In this regard, our rational design takes into consideration the supramolecular ionic PUs (SPUs) possessing both positive and negative charges will form a reversible ionic network which may result in increased mechanical, self-healing and biological properties [25]. Herein, we describe a simple catalyst-free strategy to obtain APUs 
with highly tunable mechanical properties and degradability, which can be used for engineering of blood vessels and soft connective tissues. Alginate, a natural polysaccharide that has been approved by the FDA for certain uses, contains a number of exposed carboxylate groups that can be used for ionic interactions and further functionalization, therefore, it is an ideal choice for our aim [26-28]. In contrast to previous researches concerning preparation of alginate-based PUs in hydrogel or foam states [29-33], this article has been focused on synthesis of chemically-incorporated modified alginate into the structure of stretchable PU elastomer through a chemical reaction between organic-soluble alginate salt and NCO-terminated oligourethanes. In addition, the crosslinked alginate-based materials reported by Mooney's group possess extremely low mechanical properties including a tensile strength less than $200 \mathrm{kPa}$ [12], however, we show that the mechanical properties of alginatebased PUs including Young's modulus and tensile strength of ASPUs could be increased up to $100 \mathrm{MPa}$ and $50 \mathrm{MPa}$, respectively. Furthermore, the ionic interactions between polyanionic alginate and polycationic polyurethane were used as another mechanism of toughening and strengthening to promote the mechanical properties. We envisage the covalent incorporation of polyanionic alginate into the backbone of a cationic PU to prepare dual-bonded bio-based supramolecular structures.

\section{Materials and methods}

\subsection{Materials}

Polycaprolactone (PCL) diol with an average molecular weight of 2000 g.mol ${ }^{-1}$ was obtained from Solvay Chemicals, Belgium, and dried at $60{ }^{\circ} \mathrm{C}$ under vacuum for $6 \mathrm{~h}$ before use to ensure the removal of all moisture that may interfere with isocyanate reactions. NMethyldiethanolamine (MDEA, from Merck) was dried at $100{ }^{\circ} \mathrm{C}$ for $4 \mathrm{~h}$ and used without further purification. Glacial acetic acid (HOAc), isophorone diisocyanate (IPDI) and dimethyl sulfoxide (DMSO) were purchased from Merck, Germany and purified whenever it was vital 
using $4 \AA$ molecular sieves. Sodium alginate (SA) with a molecular weight $12,000-40,000$ was purchased from Aldrich and used as received. All other chemicals were used as received from the supplier (Merck or Sigma-Aldrich).

\subsection{General procedure for synthesis of the ASPUs}

\subsubsection{Hydrophobization of sodium alginate}

In a brief, alginic acid was prepared through a simple dilute acid treatment [34]. Sodium alginate $(2 \mathrm{~g})$ was gradually added to a mixture of dilute $\mathrm{HCl}(0.6 \mathrm{~N}, 25 \mathrm{~mL})$ and $\mathrm{EtOH}$ (25 mL), and stirred overnight at $4{ }^{\circ} \mathrm{C}$. The precipitate was separated from aqueous media by filtration under vacuum and purified by repetitive washing with $\mathrm{EtOH}$ and acetone. Then, it was allowed to be dried in oven at $60{ }^{\circ} \mathrm{C}$. Afterward, a hydrophobic amine with a low molecular weight, tributylamine, was added into the aqueous dispersion of $1 \mathrm{~g}$ alginic acid under controlled-pH conditions to neutralize the carboxylic groups of alginic acid. The resulting TBA-alginate was dialyzed using a dialysis tube (MWCO, 3500) against deionized water for three days and lyophilized. The dried TBA-alginate was soluble in conventional polar organic solvents.

\subsubsection{Synthesis of alginate-free PUs}

All of the alginate-free polyurethanes were synthesized as reported previously [20]. First of all, $20 \mathrm{~g}$ of PCL diol was placed into a $250-\mathrm{cm}^{3}$ three-necked flask equipped with a magnetic stirrer, heating oil bath, and the $\mathrm{N}_{2}$ purge system. Then, desired amount of IPDI was poured into the vessel reaction for $3 \mathrm{~h}$ at $90{ }^{\circ} \mathrm{C}$ to obtain the NCO-terminated prepolymer. After that, some values of a chain extender including 1,4-BD, MDEA or DMPA was reacted with latter prepolymer to obtain final neat polyurethane was (PU 1, PU 2, and PU 7). It is important to note that all of neat PUs were synthesized under DBTDL-catalyzed conditions since the catalyst-free PUs showed inferior properties. Also the polymerization reaction of ASPUs could be self-catalyzed because of the presence of the amine-based chain extender, i.e. 
MDEA. The synthesized polyurethanes were casted onto the Teflon plate and post-cured in oven at $100{ }^{\circ} \mathrm{C}$ for $12 \mathrm{~h}$.

\subsubsection{Synthesis of alginate-based cationic and anionic PUs}

The supramolecular ionic polyurethanes (SPUs) were synthesized under external catalyst-free conditions to obtain biomaterials that were not cytotoxic. Interestingly, these materials exhibited extremely high mechanical properties compared to those DBTDL-catalyzed neat PUs. In this regard, $20 \mathrm{~g}$ of PCL diol was reacted with excess IPDI in a $250-\mathrm{cm}^{3}$ roundbottomed reactor for $3 \mathrm{~h}$ at $80{ }^{\circ} \mathrm{C}$. The resulting NCO-terminated prepolymer was mixed with desired content of MDEA to prepare a prepolymer that possessed cationic sites. Finally, the SPU networks, (PU 3 to PU 5) were achieved during the reaction of a solution of TBAalginate in DMSO $(3 \% \mathrm{w} / \mathrm{w})$ and the resulting mixture was maintained for $6 \mathrm{~h}$ at $90{ }^{\circ} \mathrm{C}$. The prepared SPU samples were casted onto the Teflon plate and dried. The alginate-based nonionic and anionic PUs were synthesized based on our recent recipe.

\subsection{Measurements}

\subsubsection{Physical and Chemical characterization of synthesized ASPUs}

The FTIR and FTIR-ATR spectra of pristine alginate, TBA-alginate, neat polyurethane and its derivatives were performed with a Bruker-Equinox 55 FTIR spectrometer (Ettlingen, Germany) which was equipped by ATR accessories. The ${ }^{1} \mathrm{H}$ NMR of modified alginate and synthesized elastomers were recorded using spectrometers of Bruker Avance (500 $\mathrm{MHz}$ ) in $\mathrm{D}_{2} \mathrm{O}$ and deuterated dimethyl sulfoxide (DMSO-D6), respectively. The chemical shifts $(\delta)$ were reported in ppm by using tetramethylsilane (TMS) as a standard. Stress-strain measurements were performed on an Instron Mechanical Testing instrument (MTS System Corporation, Eden Prairie, MN) tensile tester model 10/M at a strain rate of $50 \mathrm{~mm} \cdot \mathrm{min}^{-1}$. The test specimens, strip-like samples, with dimensions of $50 \mathrm{~mm} \times 5 \mathrm{~mm} \times$ $0.1 \mathrm{~mm}$ were cut and subjected to the mechanical analysis. The dynamic mechanical 
measurement (DMTA) was performed with a Triton, Tritec 2000 instrument over a temperature range of -100 to $100{ }^{\circ} \mathrm{C}$ at heating rate of $10{ }^{\circ} \mathrm{C} \cdot \mathrm{min}^{-1}$ and frequency of $1 \mathrm{~Hz}$. The dimensions of sample films were $30 \mathrm{~mm} \times 10 \mathrm{~mm} \times 0.1 \mathrm{~mm}$ for the DMTA measurements. The micro-structural properties of synthesized elastomers were examined by X-ray diffraction (XRD) Siemens, D5000 X-ray diffractometer at room temperature. The molecular weight (MW) of PUs was determined by gel permeation chromatography (Waters 150C ALC/GPC, with polystyrene (PS) calibration curves) in THF at $30{ }^{\circ} \mathrm{C}$. DSC analysis was recorded on a Stanton Redcroft STA-780 (London, UK). Thermogravimetric analysis (TGA) was performed using a Polymer Lab TGA-1500 instrument (London) under a $\mathrm{N}_{2}$ atmosphere from room temperature to $600{ }^{\circ} \mathrm{C}$ with a heating rate of $10{ }^{\circ} \mathrm{C} / \mathrm{min}$.

\subsubsection{Self-healing efficiency}

To obtain the PU samples with a fractured surface, random cut was made using the as-cast film. The healed samples were subjected to tensile test and the experiment was continued up to $200 \%$ strain. Healing efficiency (HE) can be defined by modulus of toughness as follows:

Healing efficiency $(\mathrm{HE} \%)=\left(\right.$ Toughness $_{\text {healed }} /$ Toughness $\left._{\text {original }}\right) \times 100$

The modulus of toughness is a measure of the area under the stress-strain curve.

\subsubsection{Water uptake}

The samples with initial weight ca. $40 \mathrm{mg}\left(\mathrm{W}_{0}\right)$ were allowed to swell in PBS $\left(37^{\circ} \mathrm{C}\right)$. After $24 \mathrm{~h}$, the excess surface water was removed using a filter paper, and swollen samples were reweighed $\left(\mathrm{W}_{\mathrm{s}}\right)$. The water uptake by the elastomers was determined by the following equation:

Hydration Degree $=\left(\mathrm{W}_{\mathrm{s}}-\mathrm{W}_{0}\right) / \mathrm{W}_{0} \times 100 \%$

\subsubsection{In vitro biodegradation assessment of elastomers}


The enzymatic degradation was performed using Pseudomonas (PS) Lipase. The experiment was carried out in enzymatic solution $\left(0.25 \mathrm{mg} \cdot \mathrm{mL}^{-1}\right.$ Lipase in PBS with $0.02 \% \mathrm{NaN}_{3}, \mathrm{pH}$ 7.4). An initial weight, $40 \mathrm{mg}$, of samples were placed into an individual bottle containing 10 $\mathrm{mL}$ PBS, and incubated with shaking at $37^{\circ} \mathrm{C}$. The samples were taken out after $12 \mathrm{~h}, 24 \mathrm{~h}$, $48 \mathrm{~h}$ and $96 \mathrm{~h}$, and rinsed carefully by deionized water. After that, the samples were dried by vacuum at $40{ }^{\circ} \mathrm{C}$ for $12 \mathrm{~h}$, and reweighed to obtain the weight loss using the following equation:

Weight loss $(\%)=\left(\mathrm{W}_{0}-\mathrm{W}_{\mathrm{t}}\right) / \mathrm{W}_{0} \times 100 \%$

where $\mathrm{W}_{0}$ and $\mathrm{W}_{\mathrm{t}}$ indicate the dry weight of the sample before and after degradation, respectively. The experiment was repeated three times and the weight loss was reported as the average of these measurements.

\subsection{Evaluation of the biological behaviors}

\subsubsection{Isolation and culture of human umbilical vein endothelial cells (HUVECs)}

Endothelial cells were obtained from human umbilical cord veins by an adaptation of the method of Jaffe et al [35]. Umbilical cords should be harvested from the placenta as soon as possible after delivery. The cord was preserved and washed in cold phosphate buffered saline (PBS, Gibco-Invitrogen). Then, veins were filled with collagenase IV $\left(0.1 \%, 1 \mathrm{mg} \cdot \mathrm{ml}^{-1}\right)$ and incubated at $37^{\circ} \mathrm{C}$ for $10 \mathrm{~min}$. The detached HUVECs were flushed out of the vein with cold PBS, collected and centrifuged for $5 \mathrm{~min}\left(4{ }^{\circ} \mathrm{C}, 1500 \mathrm{rpm}\right)$. Cell pellets were cultured in M199 medium (Gibco-Invitrogen) supplemented with 10\% FBS, 1\% NEAA, and 1\% penicillin/streptomycin. The medium was changed every two days.

\subsubsection{HUVECs characterization}

Flow cytometry

To determine cell surface marker distribution of the cells, flow cytometry analysis was used. HUVECs were trypsinized after culture. 200,000 cells per sample were used for flow 
cytometry. HUVECs were treated with antibodies according to the manufacturer's instructions. Antibodies used for flow-cytometric analysis were fluorescein isothiocyanate (FITC) or PE-conjugated antibodies against CD90 (Santa Cruz, Sc59398), CD31 (BD Pharmingen, 555445), CD45 (Dako, F0861), CD105 (R\&D Systems, FAB10971P) and isotype control IgG's. All flow cytometry data were analyzed by Flowing software (version 2.4, Turku Centre for Biotechnology) after five independent runs.

\section{Immunostaining}

Cultured HUVECs were washed with PBS and fixed by incubation for $20 \mathrm{~min}$ in $4 \%$ paraformaldehyde. The cells were permeabilized with $0.5 \%$ Triton X-100 in PBS and blocked by incubation with 5\% PBS-BSA and then, incubated overnight with primary antibodies at $4{ }^{\circ} \mathrm{C}$. Anti-mouse IgG antibodies conjugated with PE were used as secondary antibodies. Nuclei were stained with DAPI (1:1000 Sigma-Aldrich, D-8417). After each incubation, cells were washed twice with PBS. Stained cells were photographed with fluorescence microscopy (BX51; Olympus; Japan).

\subsubsection{Cell seeding and culture on elastomers}

After sterilization of elastomers by $70 \% \mathrm{EtOH}$, the samples were incubated in culture medium overnight prior to cell seeding in order to make sure for removal of surface contaminants and facilitate protein adsorption and cell attachment onto the scaffold surface. HUVECs were resuspended to a concentration of $1 \times 10^{4}$ cells $/ \mathrm{cm}^{2}$ in culture medium M-199 containing $10 \%$ fetal bovine serum (FBS). Then, the cell suspension was seeded on top of PU films in a 24-well culture plates. The medium was repeatedly replaced every 2 days; the cellular culture was maintained in an incubator at $37{ }^{\circ} \mathrm{C}$ with $5 \% \mathrm{CO}_{2}$. All experiments were run in triplicate. Cell proliferation on polymers served as reference and control substrates.

\subsubsection{In vitro biocompatibility evaluation of elastomers}


To evaluate the cell proliferation and metabolic activity of elastomers, the MTS (Promega, G5421) assay was performed according to the manufacturer's instructions. Briefly, HUVECs were seeded at a density of $1 \times 10^{4}$ cells $/ \mathrm{cm}^{2}$ on elastomers. The medium was changed every 2 days. At days 1, 3 and 7, the samples were transferred into new wells and the MTS solution was added to each well, after which the plates were incubated in the dark at $37{ }^{\circ} \mathrm{C}$ for $3 \mathrm{~h}$. The absorbance of the solution was measured at $490 \mathrm{~nm}$. The experiments were run in triplicate.

\subsubsection{Live/dead assay}

Visualization of live and dead cells was performed by staining with calcein-AM and ethidium homodimer-1 (live/dead viability kit, Invitrogen). Images were visualized using a fluorescent microscope (Olympus, BX51 with Olympus DP72 digital camera). Images were acquired using Analysis LS Starter software. All experiments were run in triplicate.

\subsubsection{In vivo biodegradation and biocompatibility of elastomers}

In this study, all experiments were performed in accordance with the guidelines established by the Committee on Animal Research at Royan Institute for Accreditation of Laboratory Animal Care. Following a short-term general anesthesia, a small transverse incision was made on the backs of the male Wistar rats that were 240-270 $\mathrm{g}$ in weight and 6-8 weeks old ( $\mathrm{n}=3$ per group). Sterile elastomers with $10 \mathrm{~mm}$ diameter and an average thickness of $0.5 \mathrm{~mm}$ were subcutaneously implanted in random positions. After surgery, the rats were allowed to recover and permitted free access to water and food. At interested time intervals $(1,4,8$ and 20 weeks post-transplantation), rats were sacrificed. The explants and surrounding tissues were dissected from each group for weight loss evaluations $(n=3)$ and histological assessments $(n=3)$. Tissue slides were prepared and stained with hematoxylin and eosin (H\&E), Masson's trichrome (MT), toluidine blue (TB) and anti CD68 marker. To determine 
the cellular events, at least three expert blinded investigators independently examined the stained tissue slides according to our previous study.

\subsubsection{Statistical analysis}

Experiments were run in triplicate for each sample. All data were expressed as mean of standard deviations. Utilizing one-way analysis of variance SPSS 16.0 software which followed by Tukey's significant difference post hoc test, statistical analysis between groups was performed. The $\mathrm{p}$ values of $<0.05$ were considered significant.

\section{Results and Discussion}

\subsection{Chemical synthesis and characterization of ASPU elastomers}

Alginate-based supramolecular ionic polyurethanes (ASPUs) were synthesized with $66.6 \%$ hard segment content through the reaction of a NCO-terminated prepolymer and a chain extender (Fig. 1a). At a glance, the composition of synthesized PUs is summarized in Fig. 1b. We chose polycaprolactone (PCL) diol (molecular weight: $2000 \mathrm{~g} / \mathrm{mol}$ ) as the soft segment of PU due to its biodegradability, and ability to crystallize. Isophorone diisocyanate (IPDI) served as the hard segment precursor for the prepolymer synthesis because of its wide use in the preparation of biocompatible and biodegradable PUs. Finally, both the low molecular weight alginate and N-methyldiethanolamine (MDEA) played a dual role in the SPU structure as both the chain extender and ionic components of the network (Supporting Information, Fig. S1). A schematic illustration of dual-linked network with covalent interactions as well as ionic interactions formed by carboxylate groups of alginate and tertiary ammonium groups of the cationic PUs is shown in Fig. 1c. In order to incorporate the alginate chemically into the PU structure, its solubility should be improved in polar aprotic organic solvents. This was performed by a simple hydrophobization process in which an equivalent ratio of tributylamine (TBA) as a low molecular hydrophobic amine was used to the carboxylate groups of alginic acid. In particular, we used tributylamine-alginate (TBA- 
Alg) for all tailor-made APUs as a chain extender in the last step of polymerization, different MDEA:alginate molar ratios in the chemical composition determined by PU 2 to PU 6, (Fig. 1a and $1 \mathrm{~b}$ ). We followed the polyaddition reaction between the modified alginate and NCOterminated cationic prepolymer (PU 4) by FTIR spectroscopy at different reaction intervals (Fig. 1d). As the reaction proceeded, there was an intensity decrease and a complete disappearance of the $\mathrm{N}=\mathrm{C}=\mathrm{O}$ stretching band at $2265 \mathrm{~cm}^{-1}$ together with the appearance of an amide I centered at $1722 \mathrm{~cm}^{-1}$ and an amide II centered at $1527 \mathrm{~cm}^{-1}$, which confirmed the success of the PU formation. We performed ${ }^{1} \mathrm{H}$ NMR spectroscopy to further verify the formation of all the synthesized PUs. In ${ }^{1} \mathrm{H}$ NMR spectra of all cases, APUs with $>98 \%$ conversion showed new peaks of protons at 4.39-5.11 ppm and 7.09-7.23 ppm [36] which were related to the backbone of alginate and the urethane groups, respectively (Supporting Information, Fig. S2-S4). These results confirmed that the isocyanate polymerization could be performed and completed in the absence of any toxic catalyst usually employed in urethane synthesis.

\subsection{Microstructural characterization of ASPUs}

By keeping the PCL crystallization and amorphous nature of alginate in mind, we hypothesized that the mechanical and microstructural properties of APUs could be easily tuned through a simple change in alginate content. We observed that various amounts of alginate affected both the degree of ionic crosslinking and the crystallinity of the resultant PU. The common PCL-based PUs demonstrated a degree of opacity due to crystallization of the PCL segment [37]. In contrast, PCL-based PUs that contained alginate in their structure were significantly more transparent suggesting that the added alginate may decrease the crystallinity of PCL and induce a disordered microstructure (Fig. 2a). This may be due to the amorphous nature of alginate as its carboxylate groups and their counterions disturbed the crystallization process of the ester segments. We confirmed the decrease in PCL 
crystallization through chemical incorporation of alginate by using XRD data (Supporting Information, Fig. S5).

Further characterization of the alginate effect on the phase transition and crystallization of PCL was performed using DSC analysis. As shown in Fig. 2b, the DSC data demonstrates excellent conformity with DMTA and XRD data. We selected 1,4-butane diol as a linear chain extender to obtain fully linear PUs to make rational investigation on the effect of MDEA possessing a methyl side group on mechanical properties of PUs. The PU sample with an ordered and fully linear structure, PU 1, demonstrated the sharpest peak in DSC corresponding to the melting of soft segment. On the other hand, the melting transition of PCL in the neat cationic PU (PU 2) was not observed at approximately $50{ }^{\circ} \mathrm{C}$ because the crystallization process of PCL and phase separation can be hindered due to the ammonium and methyl side groups of the MDEA. However, the melting transition was obvious for both PU 4 and PU 6 materials. It is important to note that in PU 4, the carboxylate groups of alginate interact with ammonium groups of cationic PU and therefore, the ester segments can easily crystallize, therefore, the area under the melting peak of PU 4 was larger than in PU 6. In this regard, all of ASPUs showed a smaller peak area and proper transparency compared to the PU 1.

The mechanical and thermal transitions detected by DMTA could be used to probe the interlocking of ionic constituents of a supramolecular network. A glance on all of mechanical and biological properties (Fig. 2-5) demonstrated that the sample PU 4, PU with equimolar ratio of MDEA and TBA-Alg (Fig. 1b), is the optimum sample. This sample shows desirable transparency, good thermal stability at elevated temperatures, the highest values of tensile strength and toughness, excellent durability in cyclic load-bearing conditions, superior selfhealing, proper biological activities and appropriate biodegradation. Fig. 2c shows the greater storage modulus of PU 4 compared to other samples in both glassy and elastomeric regions 
since the amount of ionic interactions intensified for equal molar ratios of ionic network components. The ionic crosslinking has preserved the ionic network from thermal deterioration at elevated temperatures (Supporting Information, Fig. S6) [38]. The ionic networks made by equal molar ratios of TBA-Alg and MDEA, PU 4, illustrated an extra transition compared to other samples at temperatures higher than $50{ }^{\circ} \mathrm{C}$. This transition may be due to tight ionic interactions of network components. Further investigation on viscoelastic properties of synthesized materials showed that all of ASPUs have low values of $\tan$ delta $(\tan \delta)$, less than 1, which is in the range of high performance PU elastomers (Fig. 2d). Tan delta, the ratio of loss modulus to storage modulus $\left(G^{\prime \prime} / G^{\prime}\right)$, is a measure of the material's internal friction in a defined temperature and frequency conditions. When $G^{\prime}$ is higher than $\mathrm{G}^{\prime \prime}, \tan \delta$ is $<1$, the material shows more elastic behavior than viscous properties. As shown in Fig. 2d, a broad tan delta peak obtained for the PU 2 sample indicates that this sample is fairly heterogeneous, while both PU 4 and PU 6 materials demonstrated a narrower peak and consequently, a more homogeneous microstructure. These data are in conformity with storage modulus of the PU 2 sample that indicates no detectible transition. On the other hand, the PU 4 sample possessing ionic network illustrated a lower value of tan delta compared to PU 6. This result could be simply ascribed by the presence of electrostatic interactions between ionic parts of the PU 4 network and the ability of ionic bonding rupture [12]. In order to further study the ionic network structure, we immersed the PU 4 sample into a saturated aqueous sodium chloride solution for $48 \mathrm{~h}$ after which it was subsequently analyzed again by DMTA. As shown in Fig. 2c, the increase of ionic strength in aqueous media was associated with collapse of the ionic network due to breakdown of tight ionic interactions. It was apparent that the saturated $\mathrm{NaCl}$ aqueous solution disrupted the normal pattern of SPU elastomeric behavior at moderate temperatures and confirmed the presence of ionic interactions between alginate and cationic PU. 


\subsection{Mechanical properties of ASPUs}

As shown in Fig. 2e, all of APUs, especially PU 4, demonstrated extraordinary mechanical properties. The maximum tensile stress of PU 4 was $5 \mathrm{MPa}$ and $28 \mathrm{MPa}$ greater than samples did not have a supramolecular structure (PU 6) and neat cationic PU (PU 2), respectively. It was also significantly higher (>8 MPa) than the click-based crosslinked elastomers reported by Guo et al [14]. Of interest, this significant increase in tensile stress arose from only a simple ionic attraction between a polycation and polyanion, and the polymer molecular weight cannot be fully responsible for the superior mechanical properties of ASPUs (Fig 1b, PU 4 and PU 7). In contrast to the previously reported covalently crosslinked alginate-based hydrogels with extremely low elastic modulus and low tensile strength, less than $200 \mathrm{kPa}$ [12], our ionically crosslinked ASPUs demonstrated highly robust and tunable mechanical properties. Young's modulus of APUs could be tuned from 20 to $42 \mathrm{MPa}$ for PU 2 and PU 6, respectively (Fig. 2f). This dramatic enhancement was continued in the case of Young's modulus of PU 4 up to $93 \mathrm{MPa}$, which could be directly related to the formation of a tight ionic network. It has been reported that the elasticity and plasticity of a polymeric network could be tuned by compromising the density of chemical and physical crosslinking, respectively. However, by increasing the number of secondary interactions including the electrostatic interactions between ionic parts of the network, i.e. carboxylate and ammonium groups, and the hydrogen bonding through the urethane linkages in a chemical network of polyurethane, the elasticity increases [10]. We further investigated the mechanical properties of alginate-based cationic PUs by immersion of the PU 4 material in an acidic solution $(0.1$ $\mathrm{M} \mathrm{HCl}$ ) for $24 \mathrm{~h}$ (Fig. 2f, PU 8) to support that these materials possibly have supramolecular interactions. It is obvious that all of mechanical aspects of treated sample decline because the protonation of carboxylate groups of alginate in acidic media and formation of resulting carboxylic acid groups may disturb the ionic interactions between alginate and cationic PU. 
Therefore, the polyurethanes having supramolecular interactions in the solid state exhibited much stronger stress-strain properties. The superior elongation at break of all synthesized elastomers with values of approximately $700 \%-1000 \%$, combined with the superior toughness, were other objectives that appeared to bring the unique ability for these materials to be utilized as highly stretchable and mechanically tunable elastomers.

The weakness in mechanical properties of traditional biomaterials when used in physiological wet conditions restricts their use for in vivo applications [39]. In order to assess the effect of water uptake (Supporting Information Fig. S7), we immersed the PU 6 sample, the material with the most water uptake, in PBS for $24 \mathrm{~h}$ to hydrate and subsequently analyzed its mechanical properties. Interestingly, we observed no significant differences in mechanical properties of both hydrated and dried PU 6 due to the low water uptake of PU elastomers, at most $30 \%$, which are significantly lower than common hydrogels' water uptake [5]. The small difference in mechanical properties of PU 6 material with or without water could be expectable, because the hydrophilic groups of alginate are no longer available for water uptake. In fact, both the neutralization of carboxylate groups of alginate with TBA and urethane linkages formed from the reaction of pending hydroxyl groups with isocyanate linkages making alginate much less susceptible for water uptake. Therefore, the mechanical properties of PU 6 were not significantly influenced by hydration irrespective of high alginate content in the elastomer. This negligible difference in mechanical properties for a biomaterial seems vital in dry and wet conditions because the variation in the mechanical properties of the polyesters may induce biomaterial failure and inflammatory response (Fig. 2f) [6,40].

We found that the presence of an ionic structure significantly improves both the minimal creep deformation and the rapid recovery of elongated PUs compared to their original length. To probe the mechanical stability under dynamic environments, 100 successive loadingunloading cycles were applied on the PU 4 sample. Interestingly, PU 4 exhibited a very small 
hysteresis loop (Fig. 3a), minimal loss of tensile strength and minimal creep deformation after 100 repetitive cycles compared to other samples which idealize it for load-bearing applications such as arteries. To our knowledge, this is the first report that describes a linear PU can resist a large number of cyclic stresses without significant stretching [17]. In addition to the superior fatigue-resistance, an expeditious and shape recovery of elastomers is a noteworthy aspect for those are used as load-bearing polymeric scaffolds [41].

\subsection{Self-healing properties of ASPUs}

The reversible deformability of an elastomer compared to its original length after complete failure at maximum elongation, elongation at break, is desirable, because this concept could be a measure of resilience of that material. To examine this concept, a material can be stretched up to its failure point and after that, the required time for self-recovery and the reversible deformability content are measured. As shown in Fig. 3b, the self-recovery of polymeric films formed by physical mixing of alginate and cationic PU after complete rupture is low after $60 \mathrm{~s}$, whereas the synthesized linear ASPU illustrated high reversible deformability after just $5 \mathrm{~s}$, compared to its original length due to its reversible supramolecular ionic structure. We found that the synthesized SPUs could heal intrinsically without the use of any external radiation, heat or pressure. Current chemistries that deal with self-healing properties are restricted to procedures where the healing processes are not inherently repeatable [42]. As shown in Fig. 3c, two strips of a knife-cut sample possessing a strong ionic structure, PU 4, showed rapid healing when the surfaces were brought back in conformal contact with each other. In this context, the healing efficiency of alginate-based anionic PUs was lower than that in alginate-based cationic PUs. The HE values of PU 4 were $87.3 \%$ and $70.2 \%$ for the first and third healings which are significantly higher than values of PU 7 sample (Supporting Information, Fig. S8). We repeated the healing process at most for 8 cycles with $19.4 \%$ of $\mathrm{HE}$ and found that the HE decreases down to $47.3 \%$ after 5 cycles. 
In this regard, the presence of cationic parts in PU chains and their strong affinity to carboxylate groups of alginate appeared to influence the self-healing and mechanical properties.

\subsection{Evaluation of HUVECs behavior on ASPU biomaterials}

As a measure of the cytocompatibility of PU materials, L929 cell line was used as a preliminary in vitro study. Our results demonstrated that the elastomers were not cytotoxic after $48 \mathrm{~h}$ exposure (data not shown). Given the positive preliminary data, we examined the metabolic activity of human umbilical vein endothelial cells (HUVECs, with a strongly expressed VE-cadherin at the cell-cell borders and vWF in the cytoplasm, Fig. 4a and Supporting Information, Fig. S9), using MTS assay at 1, 3 and 7 days after cell culture to assess cell behavior on related samples. The results indicated that the cellular metabolic activity continues to increase for at least 7 days (Fig. 4b). Unexpectedly, PU 6 was cytotoxic, maybe because of the large number of its exposed ammonium groups [43]. The proliferation rate of HUVECs was higher compared to PCL and similar to tissue culture polystyrene (TCPS), as the gold standard, for PU 4 at both days $1(\mathrm{p}<0.05)$ and $3(\mathrm{p}<0.01)$ (Supporting Information, Fig. S10). Moreover, Live/Dead assay demonstrated that $98 \pm 1 \%$ of HUVECs were viable after 7 days of culture on the PU 4 which was comparable with the FDAapproved PCL, $96.5 \pm 1.3 \%$ (Supporting Information, Fig. S11).

\subsection{Biodegradation evaluation of ASPU elastomers}

To explore the potential of scaffolds for use in biomedical applications, we evaluated their biodegradation profiles under in vitro and in vivo conditions. In vitro enzymatic degradation profiles of neat cationic and ASPUs in the presence of Lipase enzyme demonstrated a slower degradation compared to the PCL degradation (Fig. 4c and Supporting Information, Fig. S12a). In contrast to previous reports suggesting the urethane linkages decline the biodegradability, all of PUs have MDEA in their formulation exhibited a similar trend in 
degradation profile compared to PCL since MDEA increases the biodegradation rate [14]. Interestingly, the sample PU 6 showed substantially slower degradation profile compared to the MDEA-based PUs. Furthermore, following of PUs under in vivo degradation for 20 weeks after implantation exhibited that all of the synthesized elastomers possess comparably biodegradability similar to PCL sample. The PCL and PU 4 samples, as the FDA-approved and the elastomer with optimum properties, exhibited a remaining weight of $68.1 \pm 2.4$ and $62.0 \pm 2.9 \%$ after 20 weeks, respectively (Fig. $4 \mathrm{~d}$, and Supporting Information, Fig. S12b). No decline in degradation rate of SPU elastomers was detected compared to PCL, because in addition to increase of PCL hydrophilicity via using MDEA (Supporting Information, Fig. S7), the physical crosslinking cannot slow down the material degradation, in contrast to the chemical crosslinking [14,17].

\subsection{Biological responses of ASPU elastomers}

In order to elucidate the foreign-body reaction, we sought to investigate the in vivo cellular attraction and chronic inflammatory response. The elastomers were implanted subcutaneously in male Wistar rat. The H\&E, Masson's trichrome (MT), toluidine blue (TB) and anti-CD68 macrophage stainings were carried out to characterize the inflammatory response of implants [44]. Histological analysis of the samples at different intervals after transplantation (Fig. 5a and Supporting Information, Fig. S13 and S14) showed no adverse reactions relating to the implants could be observed during the implantation period. Following in vivo transplantation, all of the groups exhibited mild to moderate infiltration by CD68-positive macrophages. CD68-positive infiltration was characterized as minimal at 1,4 and 20 weeks post implantation. Moreover, there was a capsular layer nearby all groups of samples that filled with moderate infiltration of polymorphonuclear (PMNs), phagocytic cells (PCs) and nonphagocytic cells (NPCs) in surrounding fibroadipose tissue. Furthermore, there was a mild fibrosis in the adjacent of tissue of all samples through MT staining (Fig. 5b and Supporting 
Information, Fig. S13). Finally, new blood vessel formation was evident in supporting rat tissue during 4 and 20 weeks post-transplantation (Supporting Information, Fig. S10).

\section{Conclusion}

Alginate-based polyurethane elastomers having supramolecular interactions in their solid state were succesfully synthesized and characterized. These SPUs are highly stiff and tough, show an extensive range of high mechanical properties can be tuned by alginate content, up to $48 \mathrm{MPa}$ and $93 \mathrm{MPa}$ for tensile strength and Young's modulus respectively, and are appropriate for several biological load-bearing tissues for example vascular applications due to their incredible stability to successive loading cycles. Furthermore, we showed that this material is biocompatible, exhibites a linear biodegradation profile independent on the alginate content, and can be assumed as a novel supramolecular elastomer. 


\section{References}

[1] Yang J-A, Yeom J, Hwang BW, Hoffman AS, Hahn SK. In situ-forming injectable hydrogels for regenerative medicine. Prog Polym Sci 2014;39:1973-86

[2] Ahn BK, Lee DW, Israelachvili JN, Waite JH. Surface-initiated self-healing of polymers in aqueous media. Nat Mater 2014;13:867-72.

[3] Serrano MC, Chung EJ, Ameer GA. Advances and applications of biodegradable elastomers in regenerative medicine. Adv Funct Mater 2010;20:192-208.

[4] Annabi N, Tsang K, Mithieux SM, Nikkhah M, Ameri A, Khademhosseini A, et al. Highly elastic micropatterned hydrogel for engineering functional cardiac tissue. Adv Funct Mater 2013;23:4950-9.

[5] Chen Q, Zhu L, Zhao C, Wang Q, Zheng J. A robust, one-pot synthesis of highly mechanical and recoverable double network hydrogels using thermoreversible sol-gel polysaccharide. Adv Mater 2013;25:4171-6.

[6] Rai R, Tallawi M, Grigore A, Boccaccini AR. Synthesis, properties and biomedical applications of poly(glycerol sebacate) (PGS): A review. Prog Polym Sci 2012;37:105178.

[7] Masoumi N, Annabi N, Assmann A, Larson BL, Hjortnaes J, Alemdar N, et al. Trilayered elastomeric scaffolds for engineering heart valve leaflets. Biomaterials 2014;35:7774-85.

[8] Sharma AK, Hota PV, Matoka DJ, Fuller NJ, Jandali D, Thaker H, et al. Urinary bladder smooth muscle regeneration utilizing bone marrow derived mesenchymal stem cell seeded elastomeric poly(1,8-octanediol-co-citrate) based thin films. Biomaterials 2010;31:6207-17.

[9] Wu W, Allen RA, Wang Y. Fast degrading elastomer enables rapid remodeling of a cellfree synthetic graft into a neo-artery. Nat Med 2012;18:1148-53. 
[10] DeForest CA, Anseth KS. Cytocompatible click-based hydrogels with dynamically tunable properties through orthogonal photoconjugation and photocleavage reactions. Nat Chem. 2011;3:925-31.

[11] Shin H, Olsen BD, Khademhosseini A. The mechanical properties and cytotoxicity of cell-laden double-network hydrogels based on photocrosslinkable gelatin and gellan gum biomacromolecules. Biomaterials 2012;33:3143-52.

[12] Sun J-Y, Zhao X, Illeperuma WRK, Chaudhuri O, Oh KH, Mooney DJ, et al. Highly stretchable and tough hydrogels. Nature 2012;489:133-6.

[13] Zhu J. Bioactive modification of poly(ethylene glycol) hydrogels for tissue engineering. Biomaterials. 2010;31:4639-56.

[14] Guo J, Xie Z, Tran RT, Xie D, Jin D, Bai X, et al. Click chemistry plays a dual role in biodegradable polymer design. Adv Mater 2014;26:1906-11.

[15] Daemi H, Barikani M, Barmar M. Compatible compositions based on aqueous polyurethane dispersions and sodium alginate. Carbohydr Polym 2013;92:490-6.

[16] Heo Y, Sodano HA. Self-healing polyurethanes with shape recovery. Adv Funct Mater 2014;24:5261-8.

[17] Pereira MJN, Ouyang B, Sundback CA, Lang N, Friehs I, Mureli S, et al. A highly tunable biocompatible and multifunctional biodegradable elastomer. Adv Mater 2013;25:1209-15.

[18] Sardon H, Engler AC, Chan JMW, García JM, Coady DJ, Pascual A, et al. Organic acidcatalyzed polyurethane formation via a dual-activated mechanism: Unexpected preference of $\mathrm{N}$-activation over O-activation of isocyanates. $\mathrm{J}$ Am Chem Soc $2013 ; 135: 16235-41$. 
[19] Engels H-W, Pirkl H-G, Albers R, Albach RW, Krause J, Hoffmann A, et al. Polyurethanes: Versatile materials and sustainable problem solvers for today's challenges. Angew Chem Int Ed 2013;52:9422-41.

[20] Daemi H, Barikani M. Molecular engineering of manipulated alginate-based polyurethanes. Carbohydr Polym 2014;112:638-47.

[21] Daemi H, Barikani M, Barmar M. Highly stretchable nanoalginate based polyurethane elastomers. Carbohydr Polym 2013;95:630-6.

[22] Nohra B, Candy L, Blanco J-F, Guerin C, Raoul Y, Mouloungui Z. From petrochemical polyurethanes to biobased polyhydroxyurethanes. Macromolecules 2013;46:3771-92.

[23] Schellekens Y, Van Trimpont B, Goelen P-J, Binnemans K, Smet M, Persoons M-A, et al. Tin-free catalysts for the production of aliphatic thermoplastic polyurethanes. Green Chem 2014;16:4401-7.

[24] J Steed JW. Supramolecular gel chemistry: developments over the last decade. Chem Commun 2011;47:1379-83.

[25] Aboudzadeh MA, Muñoz ME, Santamaría A, Fernández-Berridi MJ, Irusta L, Mecerreyes D. Synthesis and rheological behavior of supramolecular ionic networks based on citric acid and aliphatic diamines. Macromolecules 2012;45:7599-606.

[26] Pawar SN, Edgar KJ. Alginate derivatization: A review of chemistry, properties and applications. Biomaterials 2012;33:3279-305.

[27] Khong TT, Aarstad OA, Skjåk-Bræk G, Draget KI, Vårum KM. Gelling concept combining chitosan and alginate—Proof of principle. Biomacromolecules 2013;14:276571.

[28] Lee KY, Mooney DJ. Alginate: Properties and biomedical applications. Prog Polym Sci 2012;37:106-26. 
[29] Travinskaya T, Savelyev YV. Aqueous polyurethane-alginate compositions: Peculiarities of behavior and performance. Eur Polym J 2006;42:388-94.

[30] Sone H, Fugetsu B, Tanaka S. Selective elimination of lead (II) ions by alginate/polyurethane composite foams. J Hazard Mater 2009;162:423-9.

[31] Zia KM, Zia F, Zuber M, Rehman S, Ahmad MN. Alginate based polyurethanes: A review of recent advances and perspective. Int J Biol Macromol 2015;79:377-87.

[32] Wang J, Ying X, Li X, Zhang W. Preparation, characterization and swelling behaviors of polyurethane-grafted calcium alginate hydrogels. Mater Lett 2014;126:263-6.

[33] Li L, Ying X, Liu J, Li X, Zhang W. Molecularly imprinted polyurethane grafted calcium alginate hydrogel with specific recognition for proteins. Mater Lett 2015;143:248-51.

[34] Daemi H, Barikani M. Synthesis and characterization of calcium alginate nanoparticles, sodium homopolymannuronate salt and its calcium nanoparticles. Sci Iran 2012;19:20238.

[35] Jaffe EA, Nachman RL, Becker CG, Minick CR. Culture of human endothelial cells derived from umbilical veins. Identification by morphologic and immunologic criteria. $\mathbf{J}$ Clin Invest 1973;52:2745.

[36] Pawar SN, Edgar KJ. Chemical modification of alginates in organic solvent systems. Biomacromolecules 2011;12:4095-103.

[37] Ma Z, Hong Y, Nelson DM, Pichamuthu JE, Leeson CE, Wagner WR. Biodegradable polyurethane ureas with variable polyester or polycarbonate soft segments: effects of crystallinity, molecular weight and composition on mechanical properties. Biomacromolecules 2011;12:3265-74.

[38] Mecerreyes D. Polymeric ionic liquids: Broadening the properties and applications of polyelectrolytes. Prog Polym Sci 2011;36:1629-48. 
[39] Calvert P. Hydrogels for soft machines. Adv Mater 2009;21:743-56.

[40] Patel A, Gaharwar AK, Iviglia G, Zhang H, Mukundan S, Mihaila SM, et al. Highly elastomeric poly (glycerol sebacate)-co-poly (ethylene glycol) amphiphilic block copolymers. Biomaterials 2013;34:3970-83.

[41] Guo M, Pitet LM, Wyss HM, Vos M, Dankers PY, Meijer E. Tough stimuli-responsive supramolecular hydrogels with hydrogen-bonding network junctions. J Am Chem Soc 2014;136:6969-77.

[42] Annabi N, Tamayol A, Uquillas JA, Akbari M, Bertassoni LE, Cha C, et al. 25th anniversary article: rational design and applications of hydrogels in regenerative medicine. Adv Mater 2014;26:85-124.

[43] Fischer D, Li Y, Ahlemeyer B, Krieglstein J, Kissel T. In vitro cytotoxicity testing of polycations: influence of polymer structure on cell viability and hemolysis. Biomaterials $2003 ; 24: 1121-31$

[44] Rajabi-Zeleti S, Jalili-Firoozinezhad S, Azarnia M, Khayyatan F, Vahdat S, Nikeghbalian S, et al. The behavior of cardiac progenitor cells on macroporous pericardium-derived scaffolds. Biomaterials 2014;35:970-82.

\section{Acknowledgements}

This study was funded by grants provided from Iran Polymer and Petrochemical Institute, Royan Institute, the Iranian Council of Stem Cell Research and Technology, the Iran National Science Foundation (INSF), and Iran Science Elites Federation. The authors also would like to appreciate Prof. David Mecerreyes (Polymat - University of the Basque Country, Spain) and Dr. Niloofar Sodeifi and Dr. Mohammad Kazemi Ashtiani (Royan Institute) for their kind comments to improve the article. 


\section{Figure captions}

Fig. 1. Chemical characterization of alginate-based polyurethane elastomers. (a) Chemical procedure used for manipulation of the supramolecular architecture. (b) Summary of feed compositions used for PU synthesis. (c) Schematic illustration of dual networks formed by alginate-based supramolecular ionic polyurethanes (ASPUs), green regions represent for the covalent linkages of urethanes between hydroxyl groups of alginate and isocyanates of NCOterminated prepolymer serve as strong bonds to maintain the shape of material and purple dash lines refer to ionic interactions between carboxylate groups of alginate and tertiary ammonium moieties of cationic PU toughen the materials by bond rupture. (d) Following the progress of the polymerization reaction between isophorone diisocyanate (IPDI) and poly( $\varepsilon$ caprolactone) (PCL) diol (step 1), NCO-terminated prepolymer with Nmethyldiethanolamine (MDEA) as step 2, and NCO-terminated cationic prepolymer with tributylamine-alginate (TBA-Alg) as step 3 at different reaction intervals by FTIR spectroscopy.

Fig. 2. Mechanical properties of alginate-based elastomers. (a) All of ASPUs were transparent (down ribbon) while neat PCL-based PU (top ribbon) was opaque. (b) DSC analysis illustrated that the ester segments of PCL can easily crystallize when the ratio of cationic and anionic parts of network is 1. (c) DMTA results revealed that the PU 4 could be stable at elevated temperatures due to the tight ionic interactions between anionic alginate and cationic PU. (d) Low values of tan delta of ASPUs is in the range of high performance PU elastomers (e) Tensile stress-strain curves of polyurethanes combined with dried and wet mechanical properties of PU 6. (f) Summary of mechanical properties for different PUs (YM: Young's modulus, UTS: ultimate tensile strength, EB: elongation at break, T: toughness).

Fig. 3. Load-bearing and self-healing properties of ASPU biomaterials. (a) 100 successive loading-unloading cycles of tensile loading on PU 4 showed that the elastomer maintains its 
dimensional stability. (b) Perfect recovery after rupture of PU 4 versus weak recovery of neat cationic PU. (c) Self-healing scheme between two freshly cut surfaces of PU 4 discloses the high affinity of dissimilar ionic components of ASPU network.

Fig. 4. In vitro and in vivo biocompatibility and biodegradation of PU samples. (a) Phasecontrast microscopic analysis of HUVECs in passage 2 in culture medium on TCPS, scale bar: $100 \mu \mathrm{m}$. (b) HUVECs metabolic activity and viability were performed on samples after 1, 3 and 7 days by MTS assay $(\mathrm{n}=3$ ) (compared to PCL, $* \mathrm{p}<0.05$ and $* * \mathrm{p}<0.01)$. (c) In vitro enzymatic degradation profiles of different PUs using Lipase enzyme. (d) In vivo biodegradation profiles of subcutaneously implanted samples at different intervals.

Fig. 5. Histological evaluation of explanted ASPUs at different intervals in vivo. (a) Representative images of H\&E, Masson's trichrome, toluidine blue and anti-CD68 stained histological sections of the subcutaneous tissue surrounding polymers. The scale bar represents $100 \mu \mathrm{m}$. (b) Characterization of foreign-body response to implants through qualitative evaluation of the inflammatory infiltrate (from 0 representing no infiltrate, to 4 representing severe infiltrate) and the fibrotic capsule content surrounding polymers. 
(a)
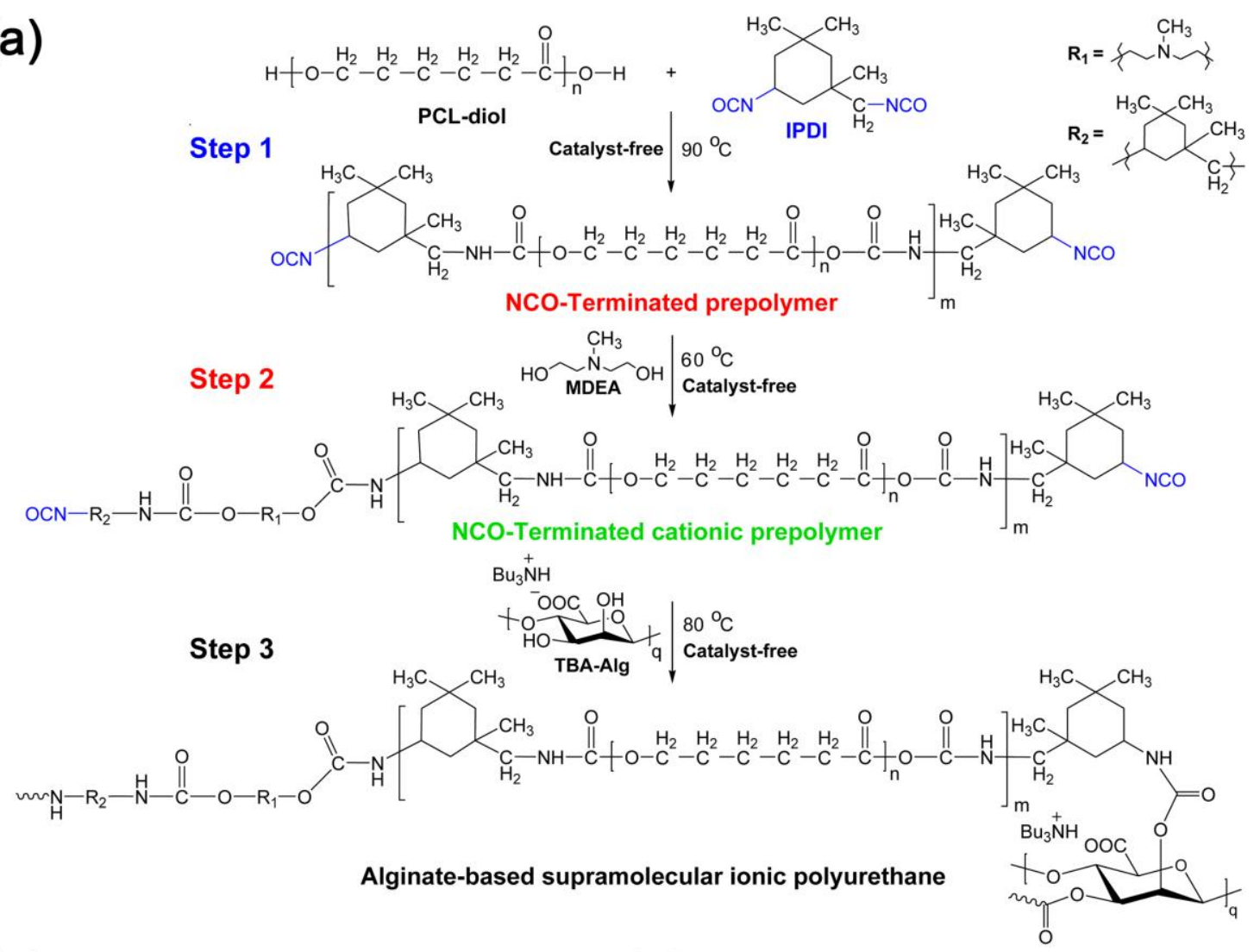

(b)

\begin{tabular}{|c|c|c|c|c|c|c|}
\hline $\begin{array}{l}\text { Sample } \\
\text { no. }\end{array}$ & $\begin{array}{l}\text { Sample } \\
\text { name }\end{array}$ & $\begin{array}{l}\text { IPDI } \\
(\mathrm{mol})\end{array}$ & $\begin{array}{l}\text { PCL diol } \\
\text { (mol) }\end{array}$ & $\begin{array}{l}\text { Chain extender } \\
\text { (mol) }\end{array}$ & $\begin{array}{l}\text { TBA-Alg } \\
(\mathrm{mol})\end{array}$ & $\begin{array}{r}M_{w} \\
k D a\end{array}$ \\
\hline PU 1 & Neat PU & 3 & 2 & 1,4-BD (1) & - & 29.4 \\
\hline PU 2 & Neat cationic PU & 3 & 2 & MDEA (1) & - & 27.9 \\
\hline PU 3 & SPU $(0.75: 0.25)$ & 3 & 2 & MDEA (0.75) & 0.25 & 47.3 \\
\hline PU 4 & SPU $(0.50: 0.50)$ & 3 & 2 & MDEA $(0.50)$ & 0.50 & 48.0 \\
\hline PU 5 & SPU $(0.25: 0.75)$ & 3 & 2 & MDEA (0.25) & 0.75 & 44.6 \\
\hline PU 6 & Alg-based PU & 3 & 2 & - & 1 & 40.3 \\
\hline PU 7 & Alg-based anionic PU & 3 & 2 & DMPA $(0.50)$ & 0.50 & 46.8 \\
\hline
\end{tabular}

(c)

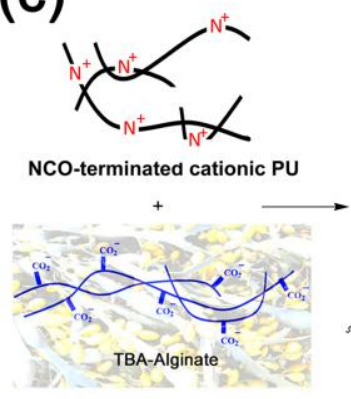

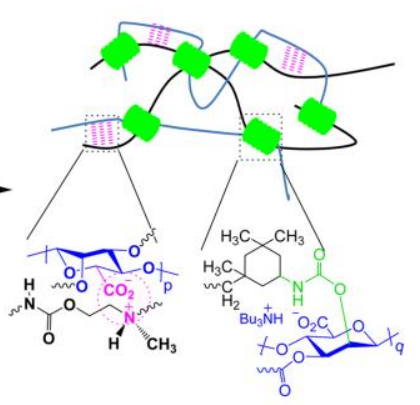

(d)

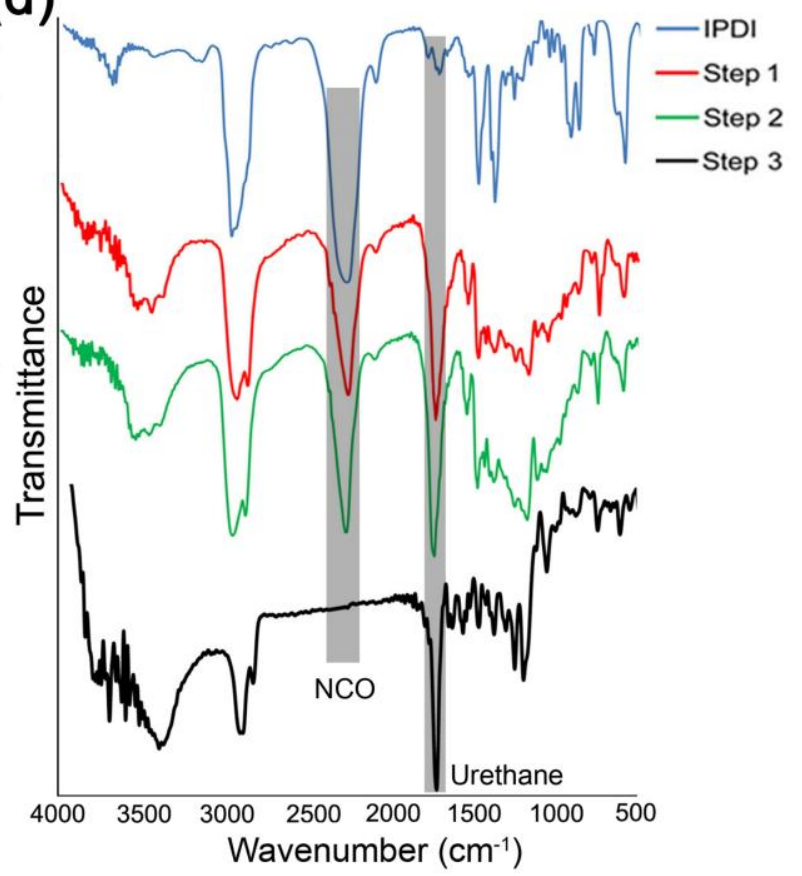

Figure 1 
(a)

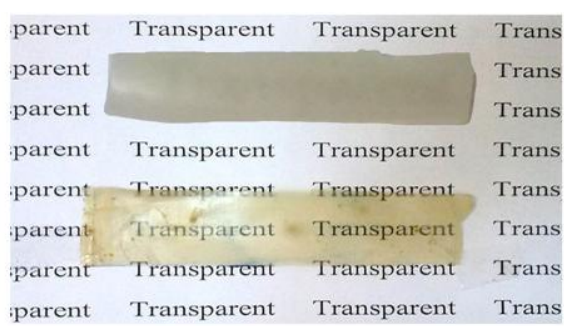

(c)

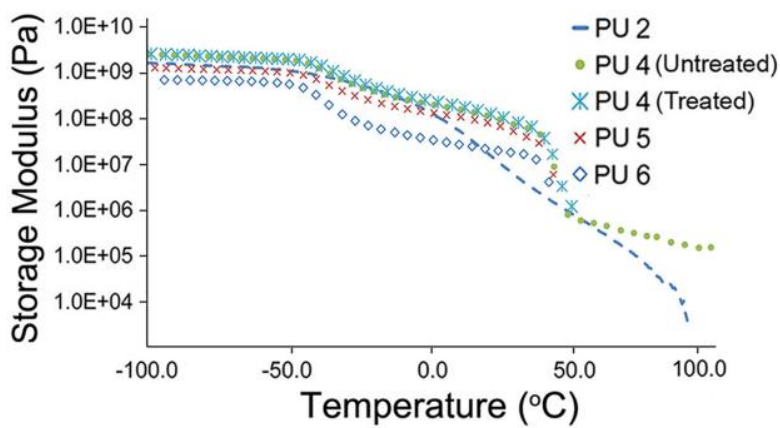

(e)

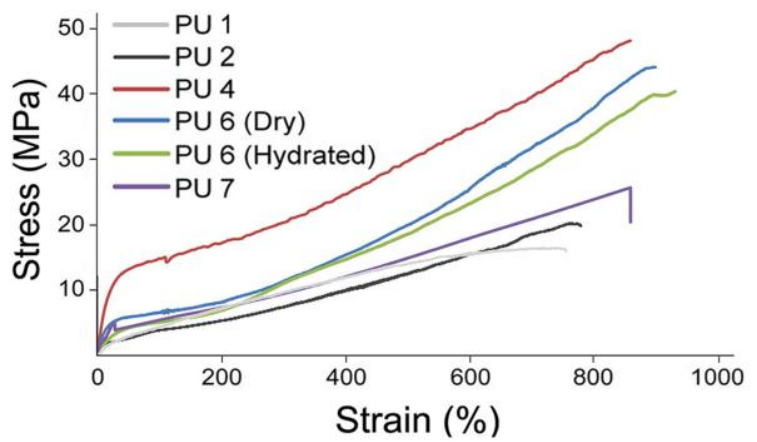

(b)

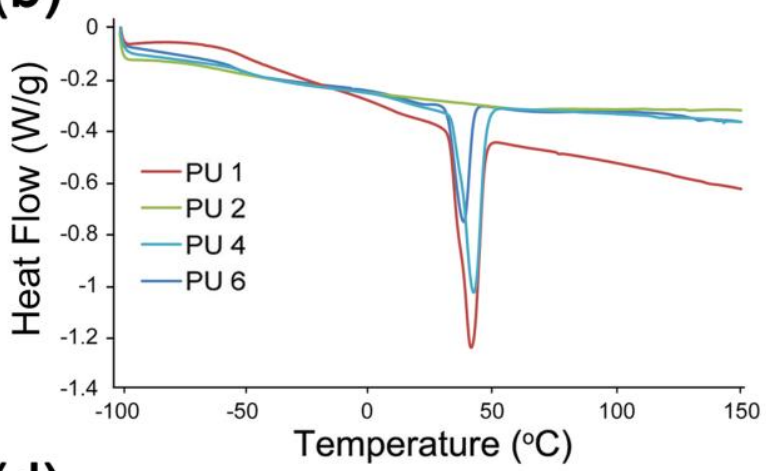

(d)

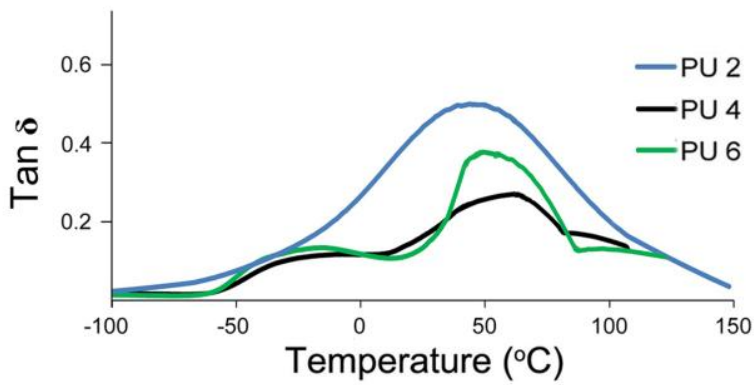

(f)

\begin{tabular}{lcccc}
\hline Sample & $\begin{array}{c}\mathrm{YM} \\
(\mathrm{MPa})\end{array}$ & $\begin{array}{c}\text { UTS } \\
(\mathrm{MPa})\end{array}$ & $\begin{array}{c}\mathrm{EB} \\
(\%)\end{array}$ & $\begin{array}{c}\mathrm{T} \\
\left(\mathrm{kJ} . \mathrm{m}^{-3}\right)\end{array}$ \\
\hline PU 1 & $14.2 \pm 1.1$ & $17.3 \pm 1.2$ & $749.7 \pm 40.5$ & $41.6 \pm 3.7$ \\
PU 2 & $15.1 \pm 0.9$ & $20.1 \pm 1.7$ & $787.4 \pm 73.7$ & $42.4 \pm 4.1$ \\
PU 3 & $52.3 \pm 1.4$ & $41.2 \pm 0.9$ & $793.7 \pm 82.3$ & $135.8 \pm 5.2$ \\
PU 4 & $\mathbf{9 2 . 8} \pm 1.6$ & $\mathbf{4 8 . 1} \pm 1.4$ & $\mathbf{8 5 9 . 5} \pm 59.1$ & $\mathbf{1 8 9 . 3} \pm 6.5$ \\
PU 5 & $56.4 \pm 1.3$ & $43.4 \pm 2.5$ & $820.4 \pm 20.3$ & $144.2 \pm 6.7$ \\
PU 6 (Dry) & $42.3 \pm 1.1$ & $44.0 \pm 1.7$ & $900.1 \pm 93.7$ & $151.2 \pm 8.3$ \\
PU 6 (Hydrated) & $33.0 \pm 0.8$ & $40.3 \pm 2.1$ & $952.9 \pm 56.5$ & $141.2 \pm 6.8$ \\
PU 7 & $18.6 \pm 1.2$ & $26.1 \pm 1.3$ & $712.3 \pm 41.8$ & $51.7 \pm 5.9$ \\
PU 8 & $38.3 \pm 1.9$ & $30.8 \pm 1.1$ & $410.9 \pm 81.8$ & $71.5 \pm 4.7$ \\
\hline
\end{tabular}

Figure 2 
(a)

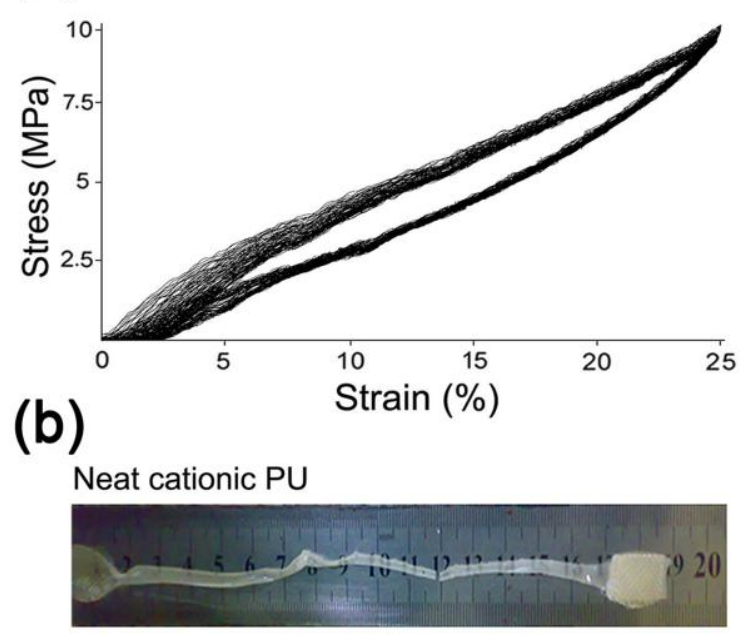

SPU (PU 4)

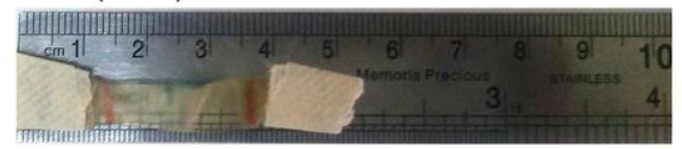

(c)
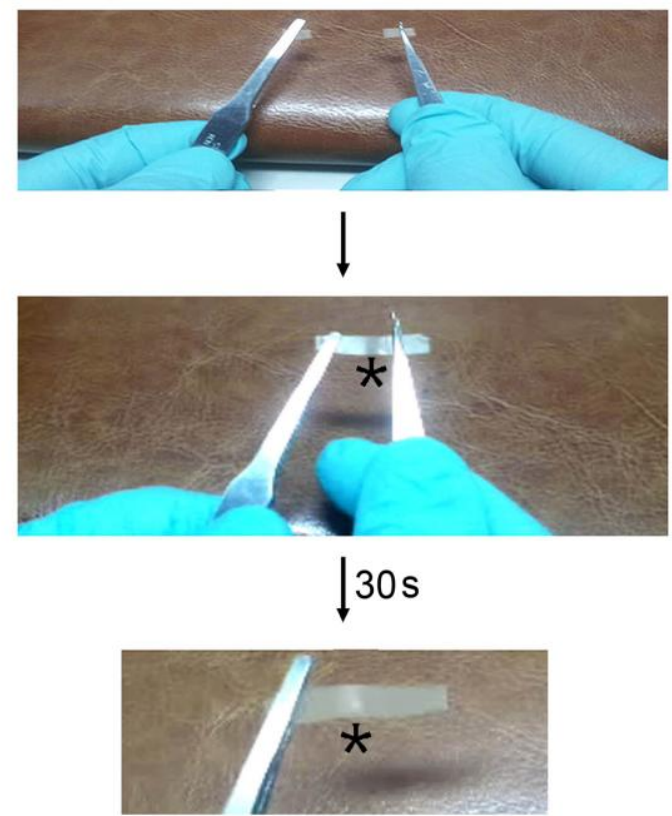

Figure 3 
(a)

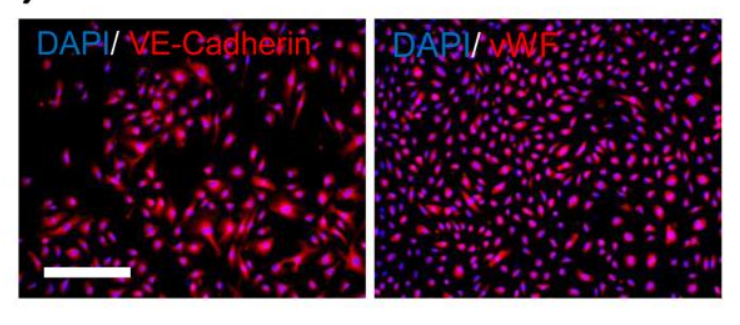

(c)

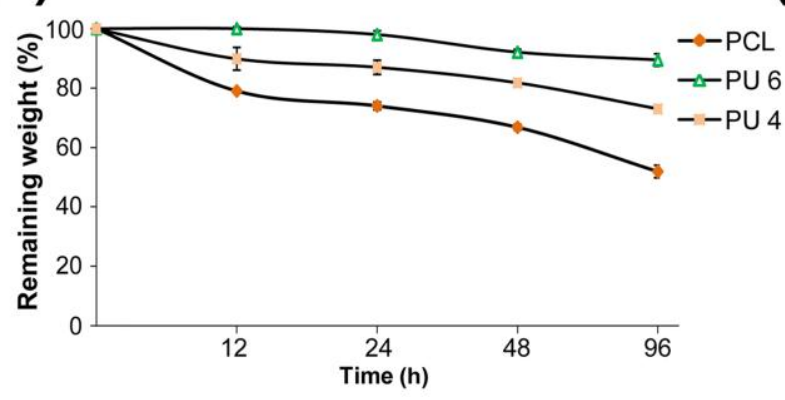

(b)

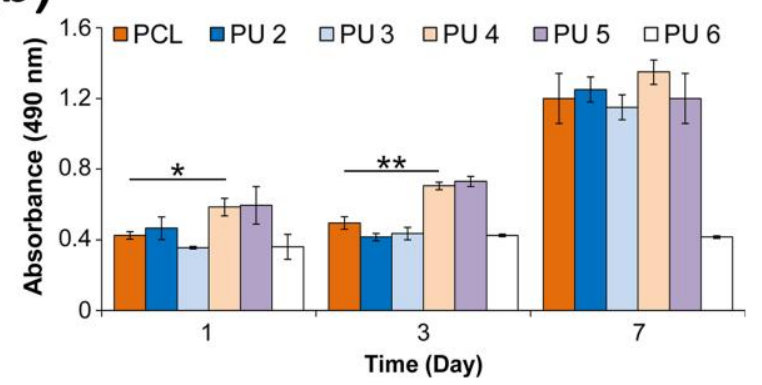

(d)

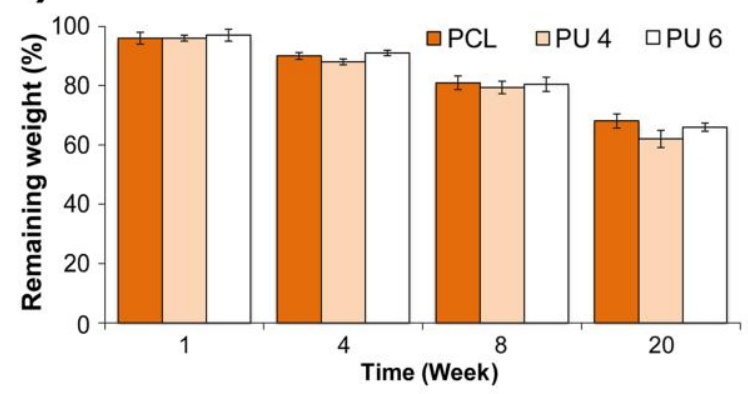

Figure 4 
(a)

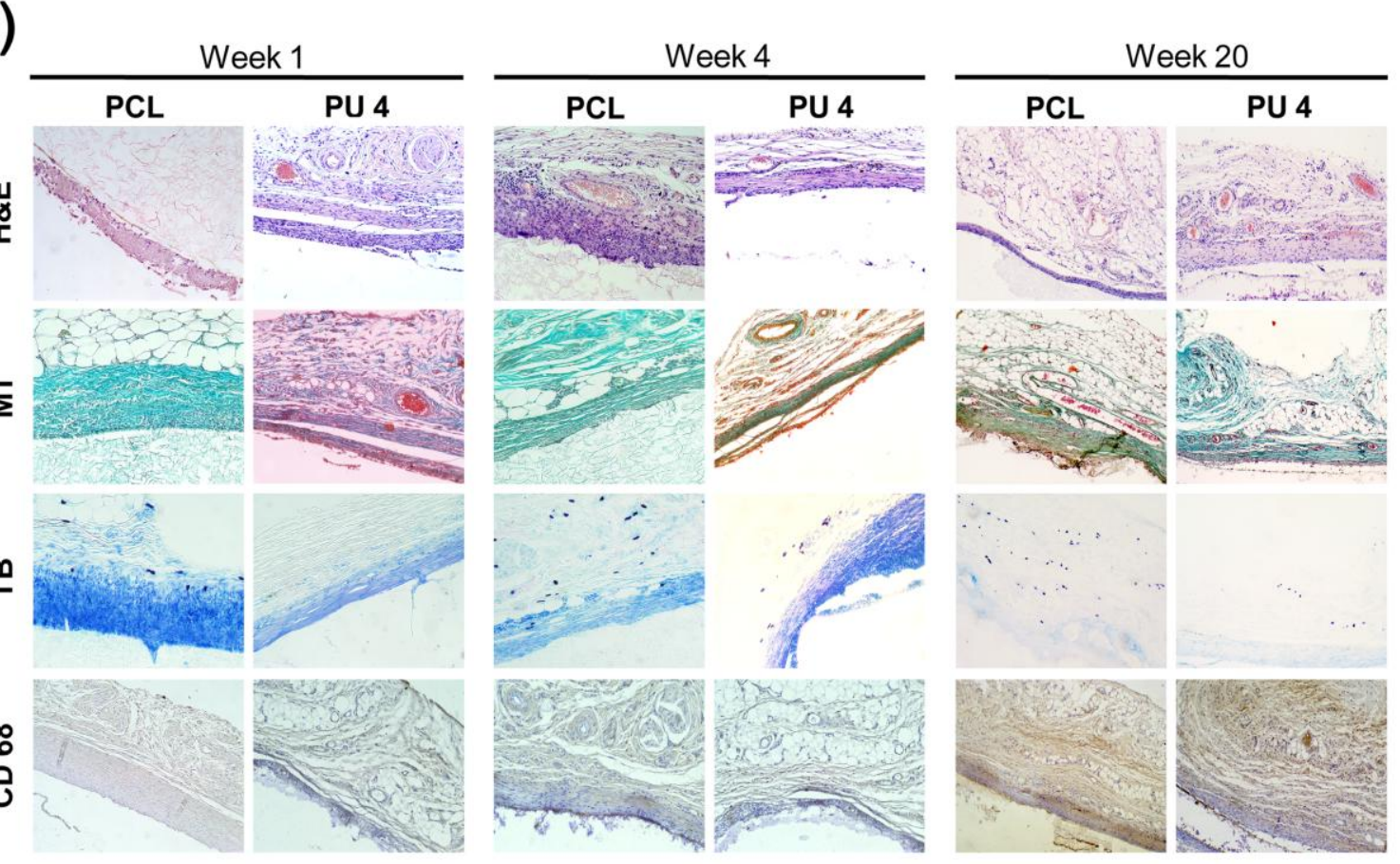

(b)
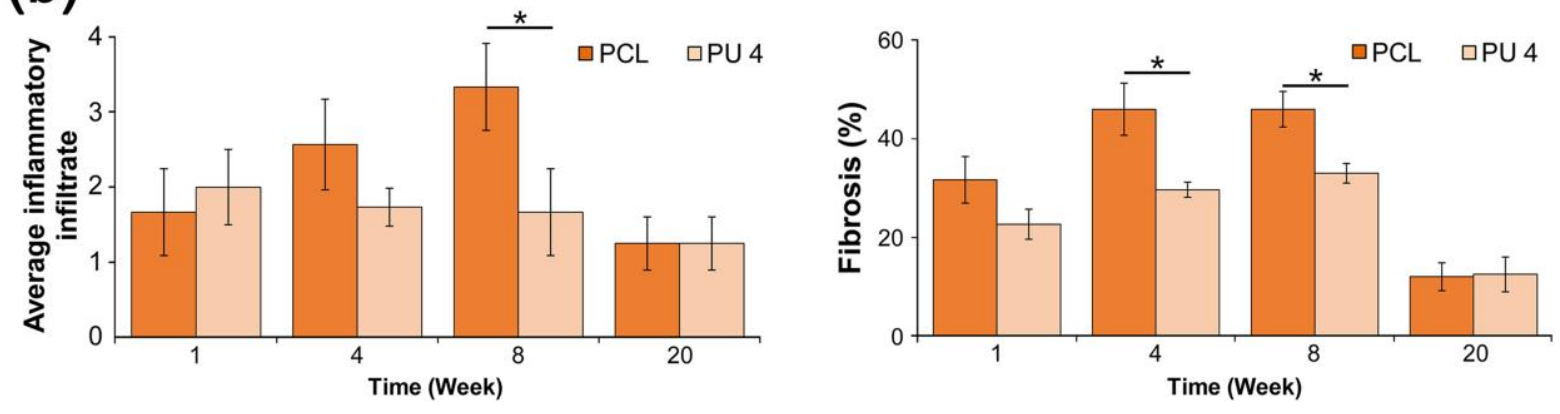

Figure 5 